\title{
Behavioral and Cognitive Behavioral Management of Anxiety
}

\author{
Lalit Pratap Singh $^{1} *$ Dr. Shikha Srivastava ${ }^{2}$
}

\section{ABSTRACT}

Understanding anxiety and depression have been the major focus of psychology over the last hundreds of years. People experience these negative psychological states as a part of their response to their threatening life events. Anxiety is a normal adaptive response to threatening situations which sometimes help to cope with threatening challenging. Humans are hard wired to response in these ways as they are adaptive subsequent to traumatic events. Anxiety is one of the most common mental health concerns in our society. They are often experienced as a complex set of emotional and functional challenges. In the daily life of people, they are exposed to stressful situations; sometimes these stressors may lead to an illnesses and mental disorders like clinically significant anxiety and other negative psychological states. The current paper will briefly describe the nature, symptoms, models and behavioral and cognitive strategies to manage anxiety. The paper will help health professionals to know these basic interventions in anxiety management and thereby improve their well-being.

Keywords: Anxiety, Management of Anxiety, Behavioral, Cognitive Behavioral Technique

Anxiety is a normal, emotional, reasonable and expected response to real or potential danger, also, it is the environment we are living in is physically, mentally, emotionally, socially and morally dynamic and challenging; we possess effective mechanisms to meet every day stress (Shri, 2010). Freud wrote extensively on anxiety. He asserted that anxiety is the base on which all psychopathology develops.

Anxiety is "a reaction to an unknown danger and it is undecided intense apprehension that is usually reflected in a characteristic combination of visceral-motor disturbances and skeletal tensions” (Rubin \& Krochak, 1988).

\section{SYMPTOMS OF ANXIETY}

Emotional symptoms: Emotional symptoms include non-stop worrying and uncontrollable anxiety. The individual is not able to stop thinking about those thoughts that cause anxiety. The individual also loses the ability to tolerate uncertainty and desperately wants to know the future.

\footnotetext{
${ }^{1}$ Research Scholar, Department of Applied Psychology, Galgotia University, Greater Noida, U.P., India

${ }^{2}$ Dept. of Applied Psychology SHSS , Galgotias University greater Noida

*Responding Author
}

Received: January 5, 2017; Revision Received: January 22, 2017; Accepted: February 2, 2017

(c) 2017 Singh L, Srivastava S; licensee IJIP. This is an Open Access Research distributed under the terms of the Creative Commons Attribution License (www.creativecommons.org/licenses/by/2.0), which permits unrestricted use, distribution, and reproduction in any Medium, provided the original work is properly cited. 


\section{Behavioral and Cognitive Behavioral Management of Anxiety}

Physical symptoms: These symptoms are physiological changes that include biological effects on the body that resulted from anxiety. Generally, these symptoms reflect elevated sympathetic autonomic nervous system activity (blood pressure, muscle tension and so on).

Behavioral symptoms: The behavioral symptoms influencing the act of the patients; they have no ability to relax, or enjoy quiet time (e.g. being easily fatigued) (Barlow, 1992).

These three types of symptoms include the following symptoms: difficulty concentrating, difficulty sleeping, irritability, fatigue/exhaustion, muscle tension repeated stomach aches or diarrhoea, sweating palms, shaking, rapid heartbeat and neurological symptoms such as complaints of numbness/tingling of different parts of the body.

\section{Models of Anxiety}

1. Incubation model: Incubation model of anxiety was given by Eysenck in the 1967.The basic idea of this model is the phenomena of extinction that is a part of classical conditioning in fact does not exist. In other words when a conditioned stimulus is presented without unconditioned stimulus for many trials, conditioned response doesn't stop; rather the strength of it rather increases. Eysenck posits that this phenomenon is also the cause of anxiety. He calls this as "Incubation".

2. Cognitive model: Cognitive theories posit that appraisal plays the pivotal role in anxiety. The appraisal process involves two stages, a primary appraisal in which an individual determines the threat posed by the environment and following a threatening primary appraisal a secondary appraisal where the individual evaluates his or her ability to cope with the demand imposed by the environment. Beck and Emery (1985) suggests that maladaptive anxiety results from distortions in the appraisal process. People who suffer from chronic anxiety are those who misperceive benign situations as threatening. Barlow (1988) extends the appraisal model and suggests that anxiety is cognitive-affective phenomena, at the core of which is negative affect. Perceptions of threat are influenced by early experiences with uncontrollability that creates a psychological vulnerability to anxiety and a biological disposition to experience anxiety in the face of negative events. Specifically, negative events; activate the biological vulnerability to stress, leading traitanxious individuals to perceive the environment as threatening even in the absence of identifiable stressor.

Meta-cognitive model: Wells (2009) developed the metacognitive model of generalized anxiety disorder (GAD). This model focuses on the role of metacognitive beliefs (i.e., thoughts about thinking) in the development and maintenance of emotional disorders and suggests that positive beliefs about the benefits of worry (e.g., "Worrying helps me cope") and negative beliefs about the danger and uncontrollability of worry (e.g., "my worrying is bad for me”) are associated with pathological worry. This model has led to the development of new treatments for 


\section{Behavioral and Cognitive Behavioral Management of Anxiety}

pathological worry, with research suggesting that modifying beliefs about worry enhances treatment outcome in anxiety disordered adults (Wells \& King, 2006).

Worry and Generalized anxiety Disorder: Worry has been described as "a chain of thoughts and images, negatively affect-laden and relatively uncontrollable’' (Borkovec et al. 1983, p. 10). Similarly, Vasey and Daleiden (1994, p. 186) describe worry as "primarily an anticipatory cognitive process involving repetitive, primarily verbal thoughts related to possible threatening outcomes and their potential consequences." Worry is also a feature of other anxiety disorders, including separation anxiety disorder and social phobia (Perrin \& Last, 1997). For example, a child with separation anxiety disorder may worry about losing or separating from his or her parent, and so may have difficulties attending school or sleeping on his or her own . Clinical worry is also associated with risk of co-morbidity with other anxiety disorders and depression.

\section{Behavioral and Cognitive-Behavioral Management of Anxiety}

Anxiety is considered as motivational force for driving behavior. It propels humans toward a specific goal. Anxiety becomes pathological when it starts impairing people's day to day functioning. For example if a person avoids going to social functions because of social anxiety, then it is a matter of concern. If a student experiences excessive anxiety before his/her exam, the academic performance is impaired. Hence, it is essential to learn to manage anxiety through psychological techniques. Here brief descriptions of behavioral and cognitive behavioral techniques are done in the following paragraphs. The techniques are described briefly in the following paragraph.

\section{Relaxation Training}

Different forms of relaxation training have been experimentally tested for decades. An early meta-analysis (Hyman et al., 1989) identified 48 experimental studies of relaxation techniques used to treat a variety of clinical symptomatology. The effect sizes ranged from 0.43 to 0.66 for the treatment of health-related symptomatology and were largest for nonsurgical samples with hypertension, headaches, and insomnia. Relaxation techniques like Jacobson Progressive Muscular Relaxation (JPMR), applied relaxation, deep breathing, pranayama etc are often used in cases of cognitive and physiological arousal conditions like anxiety, anger etc. These techniques help to reduce arousal therapy reducing anxiety. It is to be remembered that some relaxation techniques like JPMR and applied relaxation are contraindicated in patients with depression because they will further lower their arousal which might make depressed individual more depressed. In India, Rangaswami (1990) used deep relaxation training as an adjunct to anger control training with a child who exhibited uncontrolled aggression.

\section{Behavioral Activation}

The efficacy of behavioral activation was compared with cognitive therapy and antidepressant medication in a large, controlled trial with 241 adults suffering from major depression. Among 


\section{Behavioral and Cognitive Behavioral Management of Anxiety}

more severely depressed patients, behavioral activation was comparable to antidepressant medication, and both outperformed cognitive therapy (Dimidjian et al., 2006). When followed for 2 years after the initial treatment, patients receiving behavioral activation and cognitive therapy experienced similar outcomes (Dobson et al., 2008). Patients treated with medication but withdrawn onto pill-placebo suffered more relapses than patients receiving either psychotherapy. Both therapies proved less expensive and longer lasting than medication in the treatment of depression.

\section{CBT MANAGEMENT OF ANXIETY}

The roots of cognitive behaviour therapy (CBT) lie in learning and cognitive theory. The primary assumption of learning theory is that every behaviour adaptive or maladaptive is learned. Behaviors may be obseverable and covert like thoughts and subjective feelings. The learning may be direct like direct experience or indirect like observing others, reading through books, watching T.V, hearing others etc. There is a basic assumption in CBT that dysfunctional and threatening cognitions are the cause of anxiety because these cognitions directly affect emotions and behaviors. The primary goals of CBT for anxiety management are to change maladaptive learning and thought patterns. First, CBT approaches to deal with anxiety attempt to understand the roots of the presenting problem only to the degree that this understanding gives rise to a way to intervene in the "here and now." Treatment is much more focused on addressing the factors that maintain the patient's symptoms rather than understanding what gave rise to the disorder. For example, one might want to know how a parent has reacted in the past to the patient's attempts at avoidance but rather than focusing on these past interactions. This knowledge would be used to help the clinician know whether to work with parents on developing a new approach with the result of allowing for an altered learning experience for the patient.

CBT is a skills building approach. This means that clinicians are directive and sessions may appear very didactic. However, sessions are seen only as an initial step in the learning process. Meetings with the patients are used to introduce skills, provide initial practice and problemsolve; however, homework assignments outside of session provide the repeated practice required for complete skill acquisition and refinement. Moreover, given the importance of the context in which the anxious behavior occurs in behavioral theory, it necessarily follows that CBT for patient's anxiety often introduces new skills him and even other significant others who might be responsible for maintaining or causing anxiety in him or her. The patient is the major agent of change and work together with the clinician to implement the treatment. Other significant others may only act as co-therapists. In case of a child, parents and teachers are often asked to change their behavior (eg, model nonanxious self-talk), change their approach to their child's anxiety (eg, reinforce approach and provide less opportunity for avoidance), and to act as a coach for the child when he or she is completing homework assignments or generalizing skills into everyday situations. This requires a commitment on the part of the child and his or her parents that extends beyond the typical one hour per week session. 


\section{Behavioral and Cognitive Behavioral Management of Anxiety}

On the other hand, treatment is typically time-limited. Goals are set by the patients in collaboration with the therapist and, once adequate skills have been developed and treatment goals are reached, the termination process begins. In the case of most childhood anxiety disorders, treatment usually takes 12 to 16 weeks, rarely extending beyond 6 months of active treatment. However, spaced out "booster sessions" that may extend over 4 to 6 months, may be used as a way to provide review of difficult skills. This may be particularly helpful in that effective treatment may lead a child to encounter new situations because of an increase in the ability to engage in a full-range of activities. Booster sessions may be used to help a child generalize skills to these situations and ensure durability of treatment gains.

\section{COGNITIVE RESTRUCTURING}

Given the theoretical link posited by cognitive theory between erroneous or maladaptive cognitions, the subjective experience of anxiety, and anxious behavior, one of the core components of CBT for patient anxiety is cognitive restructuring of anxious cognitions. This requires the patient to first explicitly recognize their self-talk and then to understand the links between self-talk and their symptoms. Monitoring in anxiety provoking situations is often used to help to identify specific maladaptive cognitions. Restructuring may take the form of direct discussion or guided discovery to question the validity of a thought or belief. This discussion can take several forms. One basic approach is summarized in four steps. These include (1) asking informational questions to identify the thought and find data to test the veracity of the thought, (2) empathic listening, (3) summarizing, and (4) using synthesizing or analytical questions to help the child come to a new understanding.

A purely cognitive exercise may be difficult to accomplish depending on many factors. Behavioral experiments may be particularly effective methods of cognitive restructuring in initial sessions and especially with children.. Behavioral experiments can be used to target a specific cognition such as "if I ask a child to play with me, he will laugh at me." In this case, the child and therapist would design an experiment asking a peer to play with the explicit goal of testing the veracity of the child's belief. The child is asked to engage in the experiment with the explicit goal of "data collection.” Almost all of the CBT treatments for anxiety disorders use some form of cognitive restructuring. Most programs will have a component in which the child first monitors thoughts to identify those giving rise to symptoms, then actively disputes those thoughts first with the therapist and then with increasing independence, and then develops new more adaptive, coping thoughts.

\section{REPEATED EXPOSURE AND REDUCTION OF AVOIDANCE}

Exposure to feared stimuli is the central component in most CBTs for anxiety management. Early exposure therapies guided by a reciprocal inhibition hypothesis paired feared stimuli (eg, dogs, social situations, germs) with a response incompatible with anxiety often muscle relaxation. In such an approach the patient would be trained in relaxation techniques and a 


\section{Behavioral and Cognitive Behavioral Management of Anxiety}

hierarchy of feared stimuli would be developed. Systematic exposure to the feared stimuli would proceed with the patient engaging in relaxation procedures. Any symptoms of anxiety would be countered with relaxation, as the goal would be to avoid the experience of anxiety to condition an association between the once-feared stimuli and relaxation. However, such an approach has largely fallen out of favor, in part because it has been found that the relaxation training component of the treatment was often not necessary and in part because of updated theories regarding the mechanisms responsible for change in exposure therapies. Today exposure-based treatments generally have four basic phases (1) instruction, (2) hierarchy development, (3) exposure proper, and (4) generalization and maintenance.

\section{INSTRUNCTIONS}

In the instruction phase, the patient is presented with the rationale for exposure treatment. This often includes a learning-based rationale; that is, that past avoidance has been negatively reinforced with the reduction of anxiety thereby increasing the likelihood of future avoidance and escape during the peak of their fear. As such, there is little opportunity to learn the feared stimulus is in fact innocuous. A cognitive rationale emphasizing the role of increased self-efficacy and the development of more accurate and adaptive cognitions may also be included, helping the child and parent to understand that exposure without avoidance will show the child that he or she has the skills to cope with the feared situation. It is also important that the instruction phase include basic information on the understanding of fear and anxiety as many anxious patients at least implicitly expect the anxiety to increase interminably and to spiral out of control with prolonged exposure. For this reason, the patients and the significant others need to understand the nature of anxiety and that it will peak and then decrease with prolonged exposure.

\section{DEVELOPMENT OF A HIERARCHY}

Once the patient understands the rationale for exposure therapy the next step is typically to develop a graded hierarchy of feared situations that can realistically be used for exposure sessions. More specially, an exposure hierarchy consists of a series of anxiety provoking situations arranged from the least anxiety provoking to the most. It is important to make sure that enough steps are included in the hierarchy so that each step represents a gradual progression from the previous step and that the hierarchy as a whole captures all the components necessary to illicit the fear response in the patient. For example, a patient experiencing social anxiety may need to include steps in his or her hierarchy that include overt criticism to evoke an anxiety response and allow for habituation and the development of an increased perception of self-efficacy. Importantly, it may be necessary to include steps in the hierarchy that are more anxiety provoking than those the child may ever realistically be expected to face.

\section{EXPOSURE}

In this step the patient is exposed to each of the situations in the hierarchy until the anxiety dissipates. Modeling by the therapist, in which the therapist first engages in the anxiety 


\section{Behavioral and Cognitive Behavioral Management of Anxiety}

provoking task allowing the patient to watch, may precede direct engagement by the patient. Attention should be paid to both within-session habituation (eg, decrease in subjective distress or indicators of physiologic arousal) and between session habituation, as these have been found to be predictive of outcome. Exposure may be in vivo or imaginary, although in most cases in vivo exposure is generally preferred and more effective. When circumstances do not allow for in vivo exposures (eg, repeated flights for a patient with a fear of flying), virtual-reality based exposures may prove to be a useful alternative when available. During this phase, elimination of avoidance or escape behaviors is emphasized to facilitate exposure and allow the child a return to normal activities.

\section{GENERALIZATION AND MAINTENANCE}

To generalize treatment gains across situations the patient is usually given homework assignments to repeat exposures that are mastered in session across similar situations outside of the therapy room. In addition to allowing for generalization, these activities allow for solidification of the skills learned in session, and ensure that the child does not see the presence of the therapist as necessary to the control of the anxiety. Once the patient has progressed through the entire hierarchy and anxiety has significantly dissipated, planning for termination and maintenance begins. Given that anxiety and stressful situations are a normal part of life, termination should be considered when treatment goals are achieved and anxiety appears to be within normal levels for the child's developmental level. Depending on the age of the patient and other clinical issues, this phase includes giving the patient or the significant other increasing responsibility for planning exposure or cognitive restructuring exercises when new challenges present themselves. Planning for stressful situations and providing the child with written materials that can be used to reinforce and review skills after the termination of therapy can be helpful. The therapists currently may use increasingly conducting exposure sessions outside of the typical therapy context (ie, in real life situations in which the patient might expect a relapse) and providing the him/her with a physical or cognitive cue of the exposure sessions to facilitate retrieval of the non-anxious learning that took place during treatment sessions.

\section{CONCLUSIONS}

Anxiety is often considered as a perception of threat that something bad going to happen. Continuous threat and worry significantly impair one's day to day functioning. Different techniques of anxiety management are quite useful to learn to deal with threat and pressures of everyday living. Behavioral and cognitive behavioral interventions are the most common nowadays. The type of technique useful for an individual depends on his or her personality style, nature and severity of anxiety, and social support available, his/her cognitive, behavioral, affective and spiritual resources. 


\section{Behavioral and Cognitive Behavioral Management of Anxiety}

\section{Acknowledgments}

The author appreciates all those who participated in the study and helped to facilitate the research process.

Conflict of Interests: The author declared no conflict of interests.

\section{REFERENCES}

Barlow, D. H. (1992). Anxiety and its disorders: The nature and treatment of anxiety and panic. New York: Guilford.

Beck, A. T., \& Emery, G. (1985). Anxiety disorders and phobias: A cognitive perspective. New York: Basic Books.

Borkovec, T., Robinson, E., Pruzinsky, T., \& DePree, J. A. (1983). Preliminary exploration of worry: Some characteristics and processes. Behaviour Research and Therapy, 21, 9-16.

Dimidjian, S., Hollon, S. D., Dobson, K. S., et al. (2006). Randomized trial of behavioral activation, cognitive therapy, and antidepressant medication in the acute treatment of adults with major depression. Journal of Consulting and Clinical Psychology, 74, 658-670.

Dobson, K. S., Hollon, S. D., Dimidjian, S., et al. (2008). Randomized trial of behavioral activation, cognitive therapy, and antidepressant medication in the prevention of relapse and recurrence in major depression. Journal of Consulting and Clinical Psychology, 76, $468-477$.

Eysenck, H. J. (1967). A theory of the incubation of anxiety/fear responses. Behavior Research and Therapy, 6, 309-321.

Hyman, R. B., Feldman, H. R., Harris, R. B., et al. (1989). The effects of relaxation training on clinical symptoms: A meta-analysis. Nursing Research, 8, 216-220.

Perrin, S., \& Last, C. G. (1997). Worrisome thoughts in children clinically referred for anxiety disorder. Journal of Clinical Child Psychology, 26, 181-189.

Rubin, G., Slovin, M., \& Krochak, M. (1988). The psychodynamics of dental anxiety and dental phobia, Dental Clinics of North America, 33(3), 557-577.

Shri, R. (2010). Anxiety: causes and management. The Journal of Behavioral Science, 5(1), 100-118.

Vasey, M. W., \& Daleiden, E. L. (1994). Worry in children. In G. C. L. Davey \& F. Tallis (Eds.), Worrying: Perspectives on theory, assessment and treatment (pp. 185-207). Oxford, England: Wiley.

Wells, A. (2009). Metacognitive therapy for anxiety and depression. New York, NY: The Guildford Press.

Wells, A., \& King, P. (2006). Metacognitive therapy for generalized anxiety disorder: An open trial. Journal of Behavior Therapy and Experimental Psychiatry, 37, 206-212.

How to cite this article: Singh L, S Srivastava (2017), Behavioral and Cognitive Behavioral Management of Anxiety, International Journal of Indian Psychology, Volume 4, Issue 2, No. 87, ISSN:2348-5396 (e), ISSN:2349-3429 (p), DIP:18.01.047/20170402, ISBN:978-1-365-71287-6 